\title{
Directly Detecting MeV-Scale Dark Matter Via Solar Reflection
}

\author{
Haipeng An, ${ }^{1,2}$ Maxim Pospelov, ${ }^{3,4}$ Josef Pradler, ${ }^{5}$ and Adam Ritz ${ }^{3}$ \\ ${ }^{1}$ Walter Burke Institute for Theoretical Physics, California Institute of Technology, Pasadena, California 91125, USA \\ ${ }^{2}$ Department of Physics, Tsinghua University, Beijing 100084, China \\ ${ }^{3}$ Department of Physics and Astronomy, University of Victoria, Victoria, British Columbia V8P 5C2, Canada \\ ${ }^{4}$ Perimeter Institute for Theoretical Physics, Waterloo, Ontario N2J 2W9, Canada \\ ${ }^{5}$ Institute of High Energy Physics, Austrian Academy of Sciences, 1050 Vienna, Austria
}

(Received 22 August 2017; revised manuscript received 15 November 2017; published 4 April 2018)

\begin{abstract}
If dark matter (DM) particles are lighter than a few $\mathrm{MeV} / \mathrm{c}^{2}$ and can scatter off electrons, their interaction within the solar interior results in a considerable hardening of the spectrum of galactic dark matter received on Earth. For a large range of the mass versus cross section parameter space, $\left\{m_{e}, \sigma_{e}\right\}$, the "reflected" component of the DM flux is far more energetic than the end point of the ambient galactic DM energy distribution, making it detectable with existing DM detectors sensitive to an energy deposition of $10-10^{3} \mathrm{eV}$. After numerically simulating the small reflected component of the DM flux, we calculate its subsequent signal due to scattering on detector electrons, deriving new constraints on $\sigma_{e}$ in the MeV and sub-MeV range using existing data from the XENON10/100, LUX, PandaX-II, and XENON1T experiments, as well as making projections for future low threshold direct detection experiments.
\end{abstract}

DOI: 10.1103/PhysRevLett.120.141801

Introduction.-Astrophysics and cosmology provide one of the strongest arguments for an extension to the standard model (SM) of particle physics, through the need for dark matter (DM). The "theory space" for dark matter remains vast, motivating a range of experimental approaches. A well-motivated class of models achieves the required relic abundance through thermal freeze-out during the early radiation-dominated epoch, which points to particles with weak-scale interactions-weakly interacting massive particles (WIMPs) - with the required annihilation rate $\left\langle\sigma_{\text {ann }} v\right\rangle \sim 10^{-36} \mathrm{~cm}^{2}(c=1$ from now on). A range of direct detection experiments, searching for the elastic scattering of such DM particles in the galactic halo on nuclei, have now pushed the limit down to the scale of $\sigma_{n} \sim 10^{-46} \mathrm{~cm}^{2}$ for weak-scale masses [1].

Since cold DM in the halo is nonrelativisitic, detector thresholds ensure that the sensitivity weakens dramatically for masses below a few $\mathrm{GeV}$ [1-6]. In recent years, this has motivated efforts to extend this reach to lower mass scales that still allow for viable thermal relic DM candidates (see, e.g., $[7,8])$, often with interactions mediated by new light (dark) forces [9]. These efforts have included searches at colliders, fixed target proton and electron experiments, and also consideration of direct detection via electron scattering

Published by the American Physical Society under the terms of the Creative Commons Attribution 4.0 International license. Further distribution of this work must maintain attribution to the author(s) and the published article's title, journal citation, and DOI. Funded by SCOAP ${ }^{3}$.
[10-20]. The latter approach offers the possibility of extending conventional direct detection down to masses of $\sim 10 \mathrm{MeV}$ [19-21], where the halo DM kinetic energy is $E_{\mathrm{DM}}^{\text {halo }} \sim \frac{1}{2} m_{\mathrm{DM}} v^{2} \sim 5 \mathrm{eV}$. Lowering the energy threshold by $O(10)$ down to $1 \mathrm{eV}$ appears feasible [7], and there are theoretical proposals for more significant reductions (see, e.g., [22]).

In this Letter, we point out that further direct detection sensitivity to $\mathrm{DM}$ in the $10 \mathrm{keV}-10 \mathrm{MeV}$ mass range is possible through consideration of reflected DM initially scattered by more energetic electrons in the Sun (or Earth) prior to scattering in the detector. This double (or multiple) scattering trajectory allows the DM kinetic energy to be lifted to the $\mathrm{keV}$ range. Depending on the value of the reduced DM-e mass, $\mu_{\mathrm{DM}, e}$, a single scatter may result in the energy of the reflected DM,

$$
E_{\mathrm{DM}}^{\mathrm{refl}}<E_{\mathrm{DM}}^{\mathrm{refl}, \max }=\frac{4 E_{e} \mu_{\mathrm{DM}, e}}{m_{e}+m_{\mathrm{DM}}}=\frac{4 E_{e} m_{\mathrm{DM}} m_{e}}{\left(m_{e}+m_{\mathrm{DM}}\right)^{2}},
$$

being much higher than $E_{\mathrm{DM}}^{\text {halo }}$ and indeed comparable to the typical solar electron kinetic energy $E_{e} \sim k T_{e} \sim O(\mathrm{keV})$. Thus $E_{\mathrm{DM}}^{\text {refl }}$ can be above the detection threshold for a number of existing experiments, including XENON10, XENON100, LUX, PandaX-II, and XENON1T.

The basic scenario is summarized in Fig. 1. DM scattering off free electrons in the Sun generates a new (more energetic) component of the flux impinging on the Earth. While there is necessarily a geometric suppression factor, associated with rescattering in the direction of the Earth, we find that this is still sufficient to produce new 


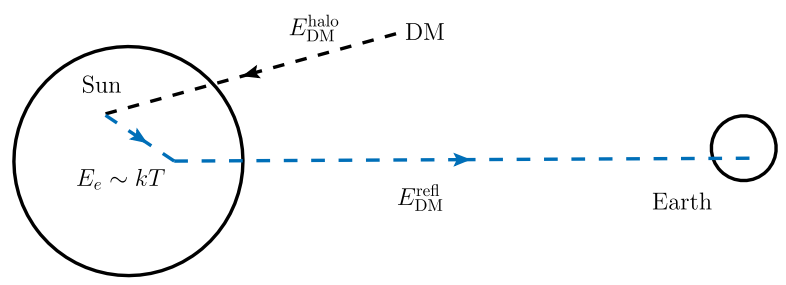

FIG. 1. A schematic illustration of the reflected dark matter flux generated through solar scattering. For bound solar electrons with energy $E_{e} \sim k T$, the DM recoil energy is bounded by the expression in Eq. (1) and can be $\sim \mathrm{keV}$.

levels of sensitivity to $\mathrm{MeV}$ and sub-MeV dark matter, where no direct detection constraints previously existed. The limits and projected sensitivity from electron scattering at a number of experiments are summarized in Fig. 2.

Solar reflection of light DM.-DM scattering on particles inside the Sun has been extensively studied as an ingredient for the indirect signature of DM annihilation to high energy neutrinos. The evolution of DM that intercepts the Sun depends crucially on its mass. Given a large enough elastic cross section on nuclei, WIMP dark matter with mass above a few $\mathrm{GeV}$ can be efficiently captured and thermalized. However, for light DM, the capture process is less efficient, and DM tends to rescatter at larger radii and evaporate. The evaporated component of the DM flux impinging on Earth may help improve sensitivity to $\sigma_{n}$ [24], and, as we are going to show, the effect mediated by $\sigma_{e}$ is even more pronounced for $\mathrm{MeV}$ and sub-MeV mass reflected $\mathrm{DM}$; for

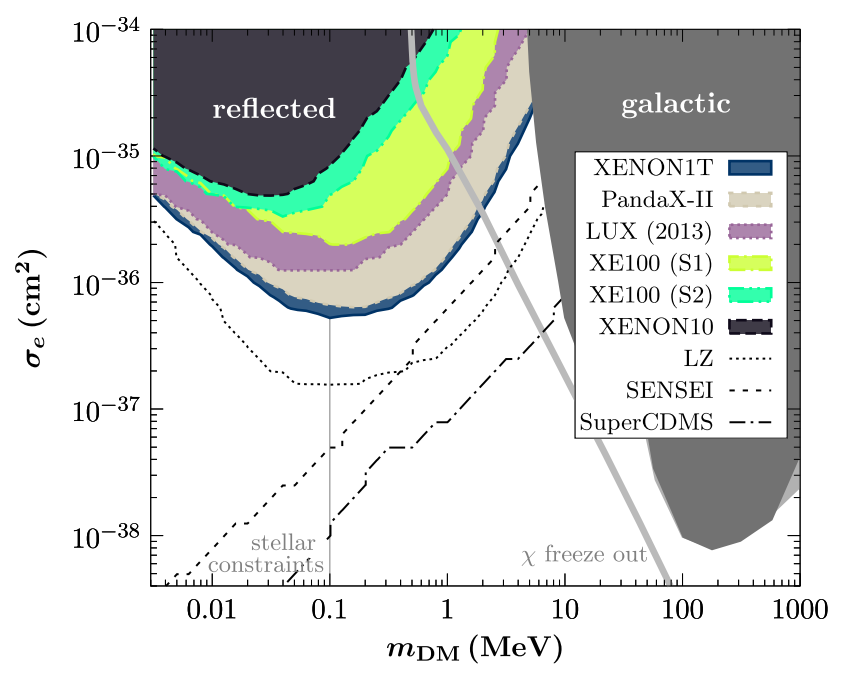

FIG. 2. Exclusion contours for reflected DM from a range of experiments are shown in comparison to previous limits from XENON10 and XENON100 on scattering from the galactic DM halo population [20,23]. Filled contours reflect current limits, while dashed contours denote future projections. The thick gray relic density contour is for the DM model in Eq. (5). A vertical line at $100 \mathrm{keV}$ indicates a schematic lower limit on $m_{\mathrm{DM}}$ from stellar energy loss while the more model-dependent cosmological $N_{\text {eff }}$ constraint is not shown (see the text). a detailed comparison between DM scattering on electrons versus nucleons inside the Sun see [25].

Depending on the scattering cross section $\sigma_{e}$, and thus the mean free path, reflection may occur after just one or two interactions, or after partial thermalization through multiple scatters within the Sun. The reflected DM flux is determined via a simulation that tracks the kinematics after initial entry into the Sun. We assume a velocityindependent $s$-wave cross section, but it is notable that the relative importance of the reflected flux would be enhanced for models with a powerlike dependence of the cross section on the relative electron-DM velocity, $\sigma_{e} \propto\left(v_{\text {rel }}\right)^{n}$, such as would occur, e.g., for scattering via higher multipoles. We note in passing that energy loss or transfer from or inside the Sun due to the scattering is negligible for the considered parameter region.

To determine the reflected contribution to the DM flux, the incoming velocity is assumed to follow a MaxwellBoltzmann distribution with an expectation value of $10^{-3}$, and an escape velocity cutoff at $2 \times 10^{-3}$. This velocity is negligible compared to solar electrons, and thus DM that scatters in the Sun acquires $E_{\mathrm{DM}}^{\text {recoil }} \sim T$. To gain some intuition, we note first that the probability of scattering off electrons in the solar core is approximately $\sigma_{e} \times R_{\text {core }} \times n_{e}^{\text {core }} \sim \sigma_{e} / \mathrm{pb}$, and thus the Sun scatters efficiently if $\sigma_{e} \gg 10^{-36} \mathrm{~cm}^{2}$. In this optically thick regime, scattering occurs in the convective zone at a characteristic radius $R_{\text {scatt }}$ given implicitly by $\sigma_{e} \int_{R_{\text {scat }}}^{R_{\odot}} n_{e}(R) d R \sim \mathcal{O}(1)$. It follows that the electron temperature, and thus the recoil energy, depends on $\sigma_{e}$, which in turn determines $R_{\text {scatt }}$, through the radius-temperature relation [26]. As the cross section is reduced, $R_{\text {scatt }}$ also decreases and $E_{\mathrm{DM}}^{\text {refl,max }}$ increases as scattering occurs in hotter regions of the core. Further decreasing the cross section ultimately increases the mean free path $\sim\left(\sigma_{e} n_{e}\right)^{-1}$ beyond the solar radius, and the strength of the reflected flux is suppressed. The scattering probability and the background DM flux in the halo, defined through the number density and average velocity as $\Phi^{\text {halo }} \equiv n_{\mathrm{DM}} v_{\mathrm{DM}}^{\text {halo }}$, may be combined into a simple estimate for the reflected DM flux incident on Earth,

$$
\Phi_{\text {refl }} \sim \frac{\Phi^{\text {halo }}}{4} \times \begin{cases}\frac{4 S_{g}}{3}\left(\frac{R_{\text {care }}}{1 \text { A.U.U }}\right)^{2} \sigma_{e} n_{e}^{\text {core }} R_{\text {core }}, & \sigma_{e} \ll 1 \mathrm{pb}, \\ S_{g}\left(\frac{R_{\text {scatt }}}{1 \text { A.U. }}\right)^{2}, & \sigma_{e} \gg 1 \mathrm{pb} .\end{cases}
$$

In the estimate (2), the overall coefficient of $1 / 4$ has a geometric origin from $\pi R_{\odot}^{2} /\left[4 \pi(1 \text { A.U. })^{2}\right] . S_{g}$ denotes the gravitational focusing effect that enhances the area at spatial infinity subtended by the effective solar scattering disk $\pi R_{\text {scatt }}^{2}$. For example, at $R_{\text {scatt }} \sim R_{\odot}$, we have $S_{g} \sim$ $1+v_{\text {esc }}^{2} /\left(v_{\mathrm{DM}}^{\text {halo }}\right)^{2} \sim O(10)$, given the value of the solar escape velocity $v_{\text {esc }}$. We note that the overall energy extracted from the Sun by reflected DM does not exceed $\sim 10 T \times \pi R_{\odot}^{2} \Phi^{\text {halo }}$, and therefore is not constrained by solar 
energetics being many orders of magnitude below solar luminosity.

Taking a representative choice of $m_{\mathrm{DM}} \sim 3 \mathrm{MeV}$, one can estimate the maximum value of the recoil energy distribution to be $\sim 0.5 T\left(R_{\text {scatt }}\right)$ at $\sigma_{e} \gg 10^{-36} \mathrm{~cm}^{2}$. For example, a single scatter would accelerate a $3 \mathrm{MeV}$ DM particle up to $\sim 100 \mathrm{eV}$ energy for $\sigma_{e} \sim 10^{-33} \mathrm{~cm}^{2}$ $\left(R_{\text {scatt }}=0.8 R_{\odot}\right)$. The reflected flux (2) in this optically thick regime is $10^{5} \mathrm{~cm}^{-2} \mathrm{~s}^{-1}$, leading to $\mathcal{O}(20)$ ionizations/ day in $1 \mathrm{~kg}$ of $\mathrm{Xe}$. This constitutes a detectable signal, and motivates a more detailed analysis.

Our preliminary estimates (2) need to be augmented to include the possibility of multiple scattering, which can significantly impact the energy of the reflected particles. Since this is difficult to treat analytically, we make use of a simulation to determine the energy spectrum and intensity of the reflected DM flux. The simulation scans the initial velocity and impact parameter to determine the initial trajectory into the Sun. The step size was chosen as $0.01 R_{\odot}$, and the standard solar model [26] was used to determine the temperature, density, and elemental abundance at each given radius. For a given cross section $\sigma_{e}$, the scattering rate was then determined probabilistically. If DM does not scatter, it propagates to the next step with velocity shifted according to the gravitational potential. If DM scatters, the electron momentum was generated according to the temperature distribution, and the new trajectory determined by first boosting to the DM-electron rest frame, and assuming an $s$-wave cross section. The gravitational effect on the trajectory was included after each nontrivial scattering. This process was repeated until the DM particle exited the Sun.

We find that it is sufficient to limit our simulations by a maximal impact parameter $\rho_{\max }=4 R_{\odot}$. Outside that range, only the slowest DM particles enter the Sun, giving a highly subdominant contribution to the reflected flux. Thus, we simulate the energy distribution $F_{A_{\rho}}(E)$ of particles interacting with (or missing) the Sun initially collected from the $A_{\rho}=16 \pi R_{\odot}^{2}$ impact area. After accounting for the gravitational redshift, $E \rightarrow E-m_{\mathrm{DM}} v_{\mathrm{esc}}^{2} / 2$, the distribution is normalized to unity, $\int_{0}^{\infty} d E F_{A_{\rho}}(E)=1$, and the resulting reflected DM flux at Earth determined via

$$
\frac{d \Phi_{\text {refl }}}{d E}=\Phi_{\text {halo }} \times \frac{A_{\rho} F_{A_{\rho}}(E)}{4 \pi(1 \text { A.U. })^{2}}
$$

As there is some arbitrariness in $A_{\rho}$, the simulated reflected flux contains an admixture of the initial unscattered distribution. This does not affect subsequent calculations because this component stays below detection thresholds.

Figure 3 shows the final kinetic energy distribution at Earth for $3 \mathrm{MeV} \mathrm{DM}$ particles. For $\sigma_{e} \sim 1 \mathrm{nb}$, the distribution turns over close to $100 \mathrm{eV}$, consistent with naive estimates. Moreover, tracking the trajectories indicates that

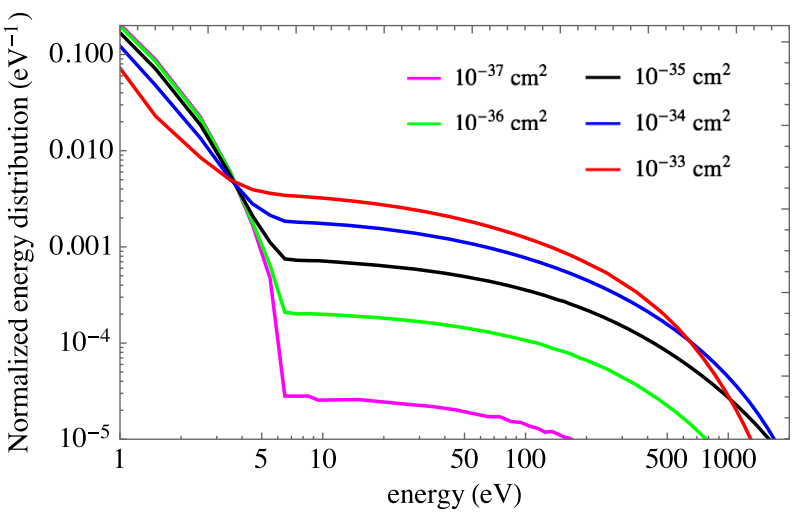

FIG. 3. Normalized energy distributions $F_{A_{\rho}=16 \pi R_{\odot}^{2}}(E)$ (in eV), are shown for reflected DM with a mass of $3 \mathrm{MeV}$ and the range of scattering cross sections indicated. The initial velocity is assumed to follow a Maxwell-Boltzmann distribution with an expectation value of $10^{-3}$, and an escape velocity cutoff at $2 \times 10^{-3}$. It is apparent that the distributions below $5-7 \mathrm{eV}$ tend to that of the background halo.

DM does indeed have a higher probability to enter the core region if the cross section is below about $10^{-34} \mathrm{~cm}^{2}$. Despite the lower cross section, the enhanced core temperature can in turn lead to fewer scatters for DM to exit the Sun, resulting in the observed enhancement in the tail of the distribution as the cross section decreases. However, the effect eventually turns off once the cross section drops well below a pb, as the mean free path and thus the collision rate becomes too low.

Direct detection via electron scattering.-With the reflected DM flux and velocity distribution in hand, the scattering signatures can be determined along the lines of the DM-electron scattering analysis of $[19,20]$, with the modifications outlined below. We consider DM scattering off bound electrons in the detector, having fixed energy $E_{e}=m_{e}-\left|E_{B}\right|$, with binding energy $E_{B}$ and a range of momenta. The process of interest corresponds to atomic ionization $\mathrm{DM}+A \rightarrow \mathrm{DM}+A^{+}+e^{-}$with $\mathrm{DM}$ threemomentum transfer $\vec{q}$. To match the literature, we write the differential scattering rate as a function of electron recoil energy in terms of a reference cross section $\sigma_{e}$ [20],

$\frac{d\left\langle\sigma_{n l} v\right\rangle}{d \ln E_{R, e}}=\frac{\sigma_{e}}{8 \mu_{\mathrm{DM}, e}^{2}} \int d q q\left|f_{n l}\left(q, p_{e}^{\prime}\right)\right|^{2}\left|F_{\mathrm{DM}}(q)\right|^{2} \eta\left(E_{\min }\right)$,

where the DM form factor $F_{\mathrm{DM}}$ can be taken to 1 if the interaction is short range. We only consider cases where the angular dependence is trivial, $q=|\vec{q}|$. The dimensionless atomic form factor describing the strength of the ionization process from atomic state $n, l$ is given by

$$
\left|f_{n l}\left(q, p_{e}^{\prime}\right)\right|^{2}=\frac{p_{e}^{\prime}}{\pi^{2} q} \int_{\left|p_{e}^{\prime}-q\right|}^{p_{e}^{\prime}+q} d p^{\prime} p^{\prime} \sum_{m=-l}^{l}\left|\left\langle\vec{p}_{e}^{\prime}\left|e^{i \vec{q} \cdot \vec{r}}\right| n l m\right\rangle\right|^{2} .
$$


We evaluate the latter using radial Hartree-Fock atomic wave functions $R_{n l}(r)$ [27] in $\psi_{n l m}(\vec{r})=R_{n l}(r) Y_{l m}(\hat{r})$ and the plane wave approximation $\left|\vec{p}_{e}{ }^{\prime}\right\rangle=e^{i \vec{p}_{e}{ }^{\prime} \cdot \vec{r}}$, including a Sommerfeld factor with effective charge $Z_{\text {eff }}=1$ [19]; $p_{e}^{\prime}=\sqrt{2 m_{e} E_{R, e}}$. When $m_{\mathrm{DM}} \ll 0.1 \mathrm{MeV}, \vec{q} \cdot \vec{r} \ll 1$ is possible. In order to avoid spurious contributions to $f_{n l}$ from potential numerical nonorthogonality in $\left\langle\vec{p}_{e}{ }^{\prime}|1| n l m\right\rangle$, we subtract the identity operator, and evaluate $\left\langle\vec{p}_{e}{ }^{\prime}\right| e^{i \vec{q} \cdot \vec{r}}-$ $1|n l m\rangle$ in these cases instead. The event rate from level $(n, l)$ is then determined by evaluating the average over the incoming energy spectrum of the reflected DM component, which in the nonrelativistic limit is $\eta\left(E_{\min }\right)=$ $\int_{E_{\min }} d E\left[m_{\mathrm{DM}} /(2 E)\right]^{1 / 2}\left(d \Phi_{\text {refl }} / d E\right) \Phi_{\text {halo }}^{-1}$. Multiplying it by the flux and target density $N_{T}$, we arrive at the total rate from the $(n, l)$ state, $d R_{n l} / d \ln E_{R, e}=N_{T} \Phi_{\text {halo }} d\left\langle\sigma_{n l} v\right\rangle /$ $d \ln E_{R, e}$, where $E_{\min }$ is the minimum DM energy required to produce an electron with $E_{R, e}$ recoil energy.

The resulting electron recoil energy spectrum is converted into scintillation $(S 1)$ and ionization $(S 2)$ responses in liquid xenon experiments, $d R_{n l} / d S i=$ $\varepsilon(S i) \int d E_{R, e} \operatorname{pdf}\left(S i \mid E_{\mathrm{dep}}^{n l}\right) d R / d \ln E_{R, e}$. Here, $\varepsilon(S i)$ is the $S i$ detection efficiency and $\operatorname{pdf}\left(S i \mid E_{\text {dep }}\right)$ is the probability to produce $S i$ given a deposited energy $E_{\text {dep }}^{n l}=E_{R, e}+\left|E_{B}^{n l}\right|$. For the purpose of this work, we consider the signals in $S 1$ and $S 2$ separately, and model $\operatorname{pdf}\left(S i \mid E_{\mathrm{dep}}^{n l}\right)$ as follows: the number of produced quanta at the interaction point is $N_{Q}=$ $E_{\mathrm{dep}} / W$ with $W=13.7 \mathrm{eV}[28,29]$, partitioned into $n_{e}$ ionized electrons escaping the interaction point and $n_{\gamma}$ scintillation photons. The latter follow a binomial distribution with $N_{Q}$ trials and single event probability $f_{e, \gamma}=$ $\left\langle n_{e, \gamma}\right\rangle / N_{Q}$. For the purpose of setting limits we only use data above $E_{\text {dep }}=0.19 \mathrm{keV}$ for computing $\left\langle n_{e}\right\rangle$, corresponding to the lowest measured charge yield [30] (together with [31]; see also [32,33]), and determine the light output self-consistently by demanding conservation of $N_{Q}$.

The detected signals are related by $N_{Q}=S 1 / g_{1}+S 2 / g_{2}$ where $g_{1}$ is the light collection efficiency and $g_{2}$ is the electron scintillation response times the electron extraction efficiency at the gas-liquid interface. For computing $S 1$ we use the respective values $g_{1}=0.12,0.1134,0.144,0.1$ $\mathrm{PE} / \gamma$ for XENON100 [34], PandaX-II (run 10) [35], XENON1T [1], and LZ [36]. For computing $S 2$ we use the respective values $g_{2}=20,12.1 \mathrm{PE} / e^{-}$for XENON100 [37] and LUX [38]; for XENON10, the data have already been converted from $S 2$ to the number of electrons [39]. $S 1$ is sampled from a binomial distribution with $n_{\gamma}$ trials and detection probability $g_{1}$; a Gaussian resolution of $\sigma_{\mathrm{PMT}} / \sqrt{\tilde{n}_{\gamma}}=0.4 \mathrm{PE}$ in detected photons $\tilde{n}_{\gamma}$ is included. For $S 2$ we assume an average $80 \%$ electron drift survival probability and apply a representative Gaussian width of $\sigma_{S 2} / \sqrt{\tilde{n}_{e}}=7 \mathrm{PE}[40]$ in the conversion of successfully drifted electrons, $\tilde{n}_{e}$, to $S 2$. After accounting for detection efficiencies, and respecting the nominal thresholds in the
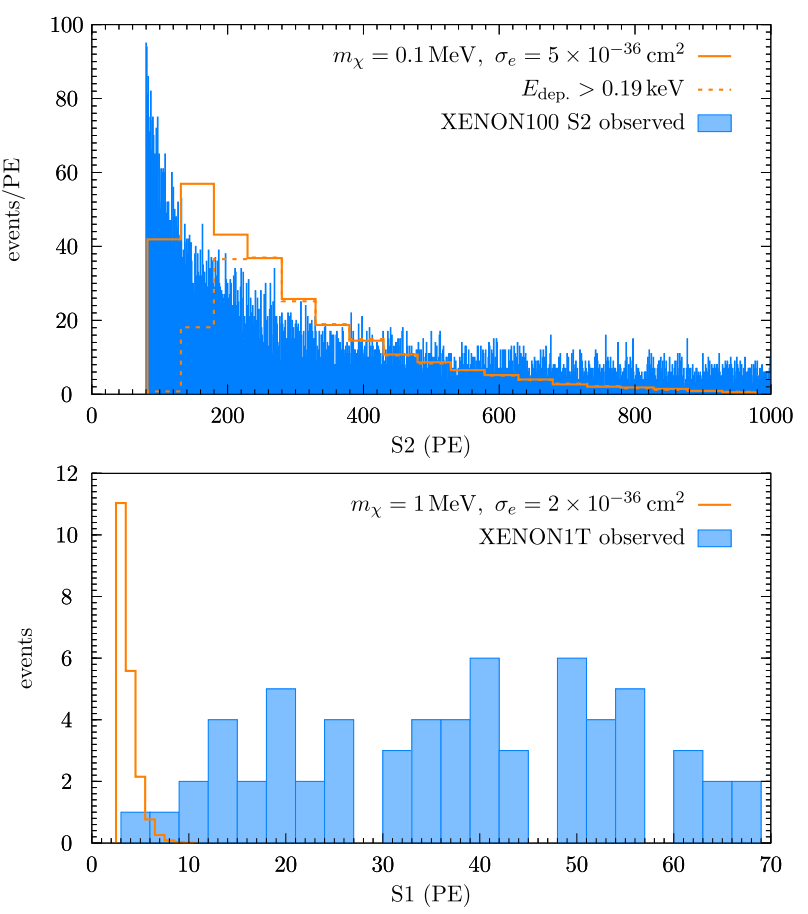

FIG. 4. Exemplary electron scattering event rates as a function of $S 2$ in XENON100 (upper panel) and as a function of $S 1$ for XENON1T (lower panel). When setting limits we require a minimum deposited energy of $E_{\mathrm{dep}}>0.19 \mathrm{keV}$ (dashed curve).

various experiments, the generated signals are compared to data as reported in $[1,3,34,38]$ and $[37,39,41]$ for $S 1$ and $S 2$ only, respectively. Exemplary spectra for $S 2$ in XENON100 and for $S 1$ in XENON1T are shown in Fig. 4. In the final step, we use the $p_{\max }$ method $[39,42]$ to arrive at the limits in the plane of $\sigma_{e}$ and $m_{\mathrm{DM}}$.

To complete this analysis, we highlight the principal reach of future direct detection experiments (making optimistic assumptions.) For LZ, the next-generation liquid xenon experiment [36], we assume, for simplicity, $100 \%$ detection efficiency in the acceptance region $S 1 \geq 3 \mathrm{PE}$ and include the solar neutrino generated background in the electron recoil band [6]. For future semiconductor experiments, we employ the ionization form factor computed in [21] and apply it to a straightforward generalization of (4); we then follow the recommendations of [21] to obtain the projections for SENSEI [43] (superCDMS [44]) with $100 \mathrm{~g} \mathrm{yr}(10 \mathrm{~kg} \mathrm{yr})$ background-free exposure and $2 e^{-}$ $\left(1 e^{-}\right)$ionization threshold. The results are summarized in Fig. 2. Further details are found in a Supplemental Material that contains additional Refs. [45-50].

Constraints on light DM models.-To demonstrate the application of our analysis, we consider a complex scalar dark matter candidate interacting with the electron vector current,

$$
\mathcal{L}_{\text {int }}=G_{\chi e} \times\left(\bar{e} \gamma^{\mu} e\right)\left(i \chi^{*} \partial_{\mu} \chi-i \chi \partial_{\mu} \chi^{*}\right)
$$


This model has been analyzed thoroughly, in particular, when the interaction is rendered UV complete via introduction of a kinetically mixed dark photon $[11,51,52]$. The $p$-wave annihilation channel allows this model to escape stringent cosmic microwave background (CMB) constraints [53]. Carrying out the standard freeze-out calculation, and adjusting the coupling in $\left\langle\sigma_{\mathrm{ann}} v_{\text {rel }}\right\rangle$ to reproduce the correct relic abundance as a function of $m_{\chi}$, we arrive at the scattering cross section given by

$$
\sigma_{e}=\frac{1}{\pi} G_{\chi e}^{2} \mu_{\chi, e}^{2} \rightarrow(8-9) \times 10^{-35} \mathrm{~cm}^{2} \times \frac{2 \mu_{\chi, e}^{2}}{\left(2 m_{\chi}^{2}+m_{e}^{2}\right) v_{e}},
$$

where $v_{e}^{2}=1-m_{e}^{2} / m_{\chi}^{2}$. When $m_{\chi}$ is close to or below $m_{e}$, a more accurate thermal average is required, which we implement numerically following Refs. [54,55]. The resulting contour is plotted in Fig. 2, and one observes that the reflected DM scattering analysis excludes the $m_{\chi}<2 \mathrm{MeV}$ region, while higher masses are currently allowed.

Going further afield in model space, there is now an increased focus on variants of the thermal relic (or WIMP) paradigm, which can ensure the correct relic abundance over the MeV mass range, e.g., SIMPs [56], ELDERS [57], and models utilizing freeze-in production with very light mediators [so that $F_{\mathrm{DM}}(q)=\left(\alpha m_{e} / q\right)^{2}$ ]. The latter case is of interest, as the target parameters correspond to $\bar{\sigma}_{e} \sim 10^{-37}-10^{-38} \mathrm{~cm}^{2}$, for $m_{\chi} \sim 100-1000 \mathrm{keV}$ [21], which provide a challenging goal for future experiments.

Discussion.-We have analyzed the direct detection sensitivity to DM-electron scattering, via an energetic reflected DM flux produced through rescattering in the Sun. This leads to new sensitivity at the sub-pb level for light dark matter in the sub-MeV mass range. Similar rescattering can also occur within Earth, which would be of particular interest in producing daily modulation. However, the up-scattering effect would be less significant due to the lower electron temperature.

The limits shown in Fig. 2 apply to all DM models with significant scattering cross sections on electrons. However, models in this mass range are subject to a number of powerful indirect constraints. Besides the CMBanisotropy-derived limits on annhilation of DM, there are constraints from stellar energy loss, and the measured radiation energy density, $N_{\text {eff }}$, as well as from primordial nucleosynthesis (BBN) [58-60]. A universally safe way of escaping the BBN and $N_{\text {eff }}$ bounds is to consider $m_{\chi}>$ few $\mathrm{MeV}$. Internally thermalized DM models with a lower mass can avoid the constraint on $N_{\text {eff }}$ (which in these models is generally shifted below 3 ), by annihilating into a mixture of SM states (e.g., photons) and neutrinolike dark radiation, as there are compensating effects on the number of equivalent neutrinos $[58,59]$. We emphasize that the new constraints derived on $\sigma_{e}$ in this paper are direct, and largely independent of additional particle content in the early Universe.
We conclude by emphasizing that reflected DM is an intrinsic contribution to the DM flux, and can be probed by all upcoming experiments with sensitivity to electron scattering, e.g., SENSEI, CRESST-III [61], SuperCDMS, LZ, and CDEX-1T [62]. We leave a study of other DM models as well as an investigation of potential signalbackground discrimination for future work [63].

H. A. is supported by the Walter Burke Institute at Caltech and by DOE Award No. DE-SC0011632. The work of M. P. and A. R. is supported in part by NSERC, Canada, and research at the Perimeter Institute is supported in part by the Government of Canada through NSERC and by the Province of Ontario through Ministry of Economic Development and Trade. J. P. is supported by the New Frontiers program of the Austrian Academy of Sciences.

[1] E. Aprile et al. (XENON), Phys. Rev. Lett. 119, 181301 (2017).

[2] D. S. Akerib et al. (LUX), Phys. Rev. Lett. 118, 021303 (2017).

[3] A. Tan et al. (PandaX-II), Phys. Rev. Lett. 117, 121303 (2016).

[4] G. Angloher et al. (CRESST), Eur. Phys. J. C 76, 25 (2016).

[5] C. Kouvaris and J. Pradler, Phys. Rev. Lett. 118, 031803 (2017).

[6] C. McCabe, Phys. Rev. D 96, 043010 (2017).

[7] M. Battaglieri et al., arXiv:1707.04591.

[8] J. Alexander et al., arXiv:1608.08632.

[9] C. Boehm and P. Fayet, Nucl. Phys. B683, 219 (2004).

[10] B. Batell, M. Pospelov, and A. Ritz, Phys. Rev. D 80, 095024 (2009).

[11] P. deNiverville, M. Pospelov, and A. Ritz, Phys. Rev. D 84, 075020 (2011).

[12] P. deNiverville, D. McKeen, and A. Ritz, Phys. Rev. D 86, 035022 (2012).

[13] J. D. Bjorken, R. Essig, P. Schuster, and N. Toro, Phys. Rev. D 80, 075018 (2009).

[14] Y. Kahn, G. Krnjaic, J. Thaler, and M. Toups, Phys. Rev. D 91, 055006 (2015).

[15] B. A. Dobrescu and C. Frugiuele, J. High Energy Phys. 02 (2015) 019.

[16] E. Izaguirre, G. Krnjaic, P. Schuster, and N. Toro, Phys. Rev. D 88, 114015 (2013).

[17] E. Izaguirre, G. Krnjaic, P. Schuster, and N. Toro, Phys. Rev. D 90, 014052 (2014).

[18] B. Batell, R. Essig, and Z. Surujon, Phys. Rev. Lett. 113, 171802 (2014).

[19] R. Essig, J. Mardon, and T. Volansky, Phys. Rev. D 85, 076007 (2012).

[20] R. Essig, A. Manalaysay, J. Mardon, P. Sorensen, and T. Volansky, Phys. Rev. Lett. 109, 021301 (2012).

[21] R. Essig, M. Fernandez-Serra, J. Mardon, A. Soto, T. Volansky, and T.-T. Yu, J. High Energy Phys. 05 (2016) 046.

[22] Y. Hochberg, Y. Zhao, and K. M. Zurek, Phys. Rev. Lett. 116, 011301 (2016).

[23] R. Essig, T. Volansky, and T.-T. Yu, Phys. Rev. D 96, 043017 (2017). 
[24] C. Kouvaris, Phys. Rev. D 92, 075001 (2015).

[25] R. Garani and S. Palomares-Ruiz, J. Cosmol. Astropart. Phys. 05 (2017) 007.

[26] J. N. Bahcall, A. M. Serenelli, and S. Basu, Astrophys. J. 621, L85 (2005).

[27] C. F. Bunge, J. A. Barrientos, and A. V. Bunge, Atomic Data Nucl. Data Tables 53, 113 (1993).

[28] C. E. Dahl, Ph.D. thesis, Princeton University, 2009.

[29] D. S. Akerib et al. (LUX), Phys. Rev. D 95, 012008 (2017).

[30] D. Huang, Ultralow Energy Calibration of LUX Detector using D-D Neutron, Tritium, and 127Xe, UCLA DM 2016, https://conferences.pa.ucla.edu/dm16/talks/huang.pdf.

[31] D. S. Akerib et al. (LUX), Phys. Rev. D 93, 072009 (2016).

[32] M. Szydagis, A. Fyhrie, D. Thorngren, and M. Tripathi, in Proceedings, Light Detection in Noble Elements (LIDINE2013), Batavia, USA, May 29-31, 2013 [JINST 8, C10003 (2013)].

[33] L. W. Goetzke, E. Aprile, M. Anthony, G. Plante, and M. Weber, Phys. Rev. D 96, 103007 (2017).

[34] E. Aprile et al. (XENON100), Phys. Rev. D 90, 062009 (2014); E. Aprile et al. (XENON100), Phys. Rev. D 95, 029904(E) (2017).

[35] X. Cui et al. (PandaX-II), Phys. Rev. Lett. 119, 181302 (2017).

[36] B. J. Mount et al., arXiv:1703.09144.

[37] E. Aprile et al. (XENON), Phys. Rev. D 94, 092001 (2016); E. Aprile et al. (XENON), Phys. Rev. D 95, 059901(E) (2017).

[38] D. S. Akerib et al. (LUX), Phys. Rev. Lett. 118, 261301 (2017).

[39] J. Angle et al. (XENON10), Phys. Rev. Lett. 107, 051301 (2011); J. Angle et al. (XENON10), Phys. Rev. Lett. 110, 249901(E) (2013).

[40] E. Aprile et al. (XENON100), J. Phys. G 41, 035201 (2014).

[41] E. Aprile, XENON1T: First Results, Patras Axion-Wimp 2017, https://indico.desy.de/indico/event/16884/session/22/ contribution/92/material/slides/0.pdf.

[42] S. Yellin, Phys. Rev. D 66, 032005 (2002).

[43] J. Tiffenberg, M. Sofo-Haro, A. Drlica-Wagner, R. Essig, Y. Guardincerri, S. Holland, T. Volansky, and T.-T. Yu, Phys. Rev. Lett. 119, 131802 (2017).

[44] R. Agnese et al. (SuperCDMS), Phys. Rev. D 95, 082002 (2017).
[45] A. Serenelli, S. Basu, J. W. Ferguson, and M. Asplund, Astrophys. J. 705, L123 (2009).

[46] E. Aprile et al. (XENON100), Astropart. Phys. 54, 11 (2014).

[47] R. F. Lang, C. McCabe, S. Reichard, M. Selvi, and I. Tamborra, Phys. Rev. D 94, 103009 (2016).

[48] M. Szydagis, N. Barry, K. Kazkaz, J. Mock, D. Stolp, M. Sweany, M. Tripathi, S. Uvarov, N. Walsh, and M. Woods, J. Instrum. 6, P10002 (2011).

[49] B. Lenardo, K. Kazkaz, A. Manalaysay, J. Mock, M. Szydagis, and M. Tripathi, IEEE Trans. Nucl. Sci. 62, 3387 (2015).

[50] K. Griest and D. Seckel, Phys. Rev. D 43, 3191 (1991).

[51] B. Holdom, Phys. Lett. 166B, 196 (1986).

[52] M. Pospelov, A. Ritz, and M. B. Voloshin, Phys. Lett. B 662, 53 (2008).

[53] T. R. Slatyer, Phys. Rev. D 93, 023527 (2016).

[54] P. Gondolo and G. Gelmini, Nucl. Phys. B360, 145 (1991).

[55] G. Belanger, F. Boudjema, A. Pukhov, and A. Semenov, Comput. Phys. Commun. 176, 367 (2007).

[56] Y. Hochberg, E. Kuflik, T. Volansky, and J. G. Wacker, Phys. Rev. Lett. 113, 171301 (2014).

[57] E. Kuflik, M. Perelstein, Nicolas Rey-Le Lorier, and Y.-D. Tsai, Phys. Rev. Lett. 116, 221302 (2016).

[58] K. M. Nollett and G. Steigman, Phys. Rev. D 89, 083508 (2014).

[59] K. M. Nollett and G. Steigman, Phys. Rev. D 91, 083505 (2015).

[60] C. Boehm, M. J. Dolan, and C. McCabe, J. Cosmol. Astropart. Phys. 08 (2013) 041.

[61] R. Strauss et al., in Proceedings, 14th International Conference on Topics in Astroparticle and Underground Physics (TAUP 2015), Torino, Italy, September 7-11, 2015; J. Phys. Conf. Ser. 718, 042048 (2016)].

[62] K.-J. Kang et al. (CDEX), Front. Phys. 8, 412 (2013).

[63] H. An, M. Pospelov, J. Pradler, and A. Ritz (to be published).

[64] See Supplemental Material at http://link.aps.org/ supplemental/10.1103/PhysRevLett.120.141801 for details on the Monte Carlo simulation of solar reflection, on the evaluation of the atomic form factor at the point of detection, on the modelling of LXE detectors, and on the exemplary DM model. 